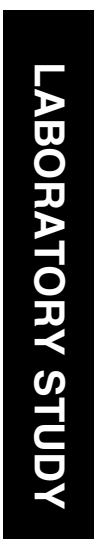

${ }^{1}$ Department of Ophthalmology, Harvard Medical School, Boston, MA, USA

${ }^{2}$ Beth Israel Deaconess Medical Center, Harvard Medical School, Boston, MA, USA

${ }^{3}$ Massachusetts Eye and Ear Infirmary, Harvard Medical School, Boston, MA, USA

${ }^{4}$ Department of Ophthalmology, Mayo

Clinic, Rochester, MN, USA

${ }^{5}$ Department of Chemistry, University of Illinois at Chicago, Chicago, IL, USA

${ }^{6}$ General Clinical Research Center and Biometrics, Harvard Medical School, Boston, MA, USA

${ }^{7}$ Retina Consultants, Charleston, WV, USA

*Correspondence: Dr JG Arroyo,

Division of Ophthalmology, Retina Service,

Beth Israel Deaconess Medical Center, Shapiro 5th floor, 330 Brookline Avenue, Boston, MA 02215, USA Tel: + 16176673391 ; Fax: + 16176675025 . E-mail: jarroyo@

bidmc.harvard.edu

Received: 11 April 2007 Accepted in revised form: 4 September 2007

Published online:

19 October 2007

\section{Amino-acid levels in subretinal and vitreous fluid of patients with retinal detachment}

\author{
Abstract \\ Purpose To compare the concentration of \\ amino acids in subretinal and vitreous fluid of \\ patients with primary rhegmatogenous retinal \\ detachment to that of control vitreous. \\ Methods This prospective, observational \\ study measured amino-acid levels in \\ subretinal fluid of patients undergoing scleral \\ buckle placement $(n=20)$ and vitreous fluid in \\ patients undergoing pars plana vitrectomy \\ $(n=5)$ for primary retinal detachment. \\ Vitreous fluid from patients undergoing \\ vitrectomy for macular hole $(n=7)$ or \\ epiretinal membrane $(n=3)$ served as a \\ control. Subretinal fluid and control vitreous \\ were analysed using high-pressure liquid \\ chromatography. Retinal detachment \\ vitreous was analysed using capillary \\ electrophoresis-laser-induced fluorescence. \\ Results Mean levels of glutamate \\ $(27.0 \pm 1.7 \mu \mathrm{M})$, aspartate $(4.1 \pm 4.0 \mu \mathrm{M})$, and \\ glycine $(44.1 \pm 31.0 \mu \mathrm{M})$ in subretinal fluid and \\ glutamate $(13.4 \pm 11.9 \mu \mathrm{M})$ in the vitreous were \\ significantly elevated in retinal detachment \\ compared to control vitreous. A significant, \\ positive association was observed between \\ levels of aspartate and glutamate in subretinal \\ fluid (Spearman's correlation coefficient: 0.74, \\ $P<0.01$ ). Mean arginine levels did not differ \\ significantly between subretinal fluid and \\ control vitreous. Levels of alanine, tyrosine, \\ valine, isoleucine, leucine, and phenylalanine \\ were significantly lower in subretinal fluid \\ compared to control vitreous (all $P<0.01$ ). \\ Conclusions Glutamate levels in subretinal \\ fluid and vitreous of patients with primary \\ retinal detachment is significantly elevated in \\ comparison to control vitreous. This finding \\ lends further support to the hypothesis that \\ elevated glutamate levels may result from \\ ischaemia of the outer retina secondary to \\ retinal detachment.
}

KM Bertram ${ }^{1,2}$, DV Bula1,2,3, JS Pulido4, SA Shippy ${ }^{5}$, S Gautam²,6, M-J Lu', RM Hatfield7, J-H Kim ${ }^{1,2}$, MT Quirk ${ }^{1,2}$ and JG Arroyo ${ }^{1,2,3 *}$

Eye (2008) 22, 582-589; doi:10.1038/sj.eye.6702993; published online 19 October 2007

Keywords: amino acids; glutamate; retinal detachment; subretinal fluid

\section{Introduction}

Patients who undergo retinal detachment (RD) repair for a macula-on ${ }^{1}$ or macula-off $\mathrm{RD}^{2}$ often lose vision despite a good anatomic result. The mechanisms underlying this vision loss are not fully understood. Many abnormal molecular and cellular events occur rapidly after RD and could be responsible for this visual impairment. Photoreceptor outer segment degeneration appears to be an initial cause of vision loss following RD. ${ }^{3-6}$ Studies in detached cat retina show chronic changes in this area that include formation of membrane-bound sacs and atrophy. ${ }^{3}$ While reattachment may induce rod and cone outer segments to regenerate, a primate study showed persistent abnormalities such as altered disc stacking in this region of the retina. ${ }^{7}$ Other events affecting the

photoreceptors include apoptosis and loss of structural integrity, both of which are thought to contribute to the vision impairment following RD. ${ }^{8-10}$ Misalignment of photoreceptors, measured by Stiles-Crawford function, may also be a problem after RD repair. ${ }^{11,12}$ Müller cells have been shown to react within $15 \mathrm{~min}$ of $\mathrm{RD},{ }^{13}$ with proliferation and hypertrophy of their processes seen as little as two days postdetachment. ${ }^{14,15}$ This abnormal growth leads to their extension into the subretinal space, formation of intraretinal scars, and irregularities within retinal synaptic connections. ${ }^{14,16,17}$ Visual recovery also may be hampered by an outgrowth of neurites from second- (bipolar and horizontal cells) and third-order neurons 
(ganglion cells). This effect, termed sprouting, may contribute to unstable retinal circuitry after RD. ${ }^{18,19}$ Elevated levels of glutamate have been shown to be toxic to retinal neurons, especially retinal ganglion cells. ${ }^{20,21}$ Animal studies have shown that alterations within the glutamatergic system of the retina occur early and could be one of the first biochemical changes associated with RD. ${ }^{22}$ Excitotoxicity produced by glutamate induces degeneration in the inner retina ${ }^{23}$ and could be a mediating factor in suboptimal vision following RD.

The separation of the neural retina from the underlying choroidal vasculature creates an ischaemic environment in the outer retina, which can induce pathological metabolic and cellular alterations. ${ }^{10,24,25}$ Accumulation of glutamate has been observed in animal models of retinal ischaemia, ${ }^{26-29}$ and animal studies of RD have shown excess glutamate levels and lower glutamine synthetase (GS) activity within the retina. ${ }^{30-32}$ Moreover, increased levels of glutamate were recently found in the vitreous fluid of human subjects with RD. ${ }^{33}$

It was hypothesized that the retinal ischaemia associated with RD leads to the accumulation of glutamate within the retina. To test this hypothesis, the level of glutamate and other amino acids in subretinal and vitreous fluid of patients with primary RD was measured and compared to that in control vitreous. The control vitreous used in this study was taken from patients undergoing retinal surgery for either epiretinal membrane or macular hole. We are unaware of any previous study that has examined amino-acid levels in subretinal fibrosis (SRF) of patients with primary RD.

\section{Methods}

\section{Patients}

This is a prospective, observational study of amino-acid levels in SRF of patients undergoing a scleral buckle placement and vitreous fluid in patients undergoing pars plana vitrectomy for primary RD. The study protocol and consent form was approved by the Human Studies Committee at the Massachusetts Eye and Ear Infirmary. None of the patients in this study had had previous retinal surgery. Patients undergoing vitrectomy surgery for macular hole or epiretinal membrane served as controls. Demographic information including age, sex, and affected eye was collected for each study group. Relevant clinical characteristics were recorded for later statistical analysis. Patients were specifically questioned to estimate the duration between occurrence of the RD and time of sampling. Snellen visual acuity was recorded at the first visit and last follow-up visit and later converted into the logarithm of the minimal angle of resolution $(\log M A R)$ units. LogMAR units was calculated by obtaining the logarithm of the reciprocal of the Snellen visual acuity for vision better than or equal to $20 / 400$. The following conversion was used for patients with vision worse than 20/400: counting fingers $=1.6$, hand movements $=2.0$, light perception $=2.5$, and no light perception $=3.0 \log \mathrm{MAR}$ units.

\section{Sample collection}

Subretinal fluid samples were obtained from 20 patients undergoing scleral buckle placement for $\mathrm{RD}$ via modified external needle drainage. ${ }^{34}$ In this procedure, the intraocular pressure is temporarily raised to high levels by tightening the scleral buckle. The subretinal space is entered externally, under the bed of the buckle, using a $27 \mathrm{G}$ needle on a $3 \mathrm{~cm}^{3}$ syringe without the plunger. The procedure is directly visualized ophthalmoscopically and the SRF is removed passively. As the drainage proceeds, the needle tip is usually redirected in the subretinal space to remove remaining SRF and to avoid retinal perforation. Once the SRF drainage is complete, the needle is withdrawn from the eye and the area is inspected. This method of collecting SRF provides relatively clean samples, free from contamination with blood. In this study, SRF samples were immediately placed on dry ice and frozen at $-80^{\circ} \mathrm{C}$ until amino acid analysis. Undiluted vitreous specimens $(\sim 1.5 \mathrm{ml})$ from 5 patients with RD and 10 patients with either an epiretinal membrane $(n=7)$ or macular hole $(n=3)$ undergoing pars plana vitrectomy were collected. Vitreous fluid was obtained via a $3 \mathrm{~cm}^{3}$ syringe that was connected to a three-way stopcock system that had been placed in the aspiration line near the vitreous cutter. ${ }^{35}$ Prior to turning on the infusion, the stopcock lever is directed away from the vitrectomy machine and the vitrectomy cut rate is lowered to 250 cuts per minute. Vitreous was then aspirated using manual aspiration. After the vitreous sample was obtained, the stopcock lever was returned to its original position and the syringe was removed. For RD-Vit samples, the tube contained $100 \mu \mathrm{l} 1 \mathrm{M}$ acetic acid ( $\mathrm{pH} 2-3$ ) to deactivate protease. The samples were placed on dry ice and immediately stored at $-80^{\circ} \mathrm{C}$ (SRF and Control-Vit) or $4^{\circ} \mathrm{C}$ (RD-Vit) until amino-acid analysis.

\section{Amino-acid analysis}

Before being sent for analysis, the samples were given a unique code for identification. Subretinal fluid and vitreous fluid controls were analysed for 10 amino acids using a Beckman 7300 Amino Acid Analyzer: alanine (Ala), arginine (Arg), aspartate (Asp), glutamate (Glu), glycine (Gly), isoleucine (Ile), leucine (Leu), phenylalanine (Phe), tyrosine (Tyr), and valine (Val). The Beckman analyzer uses an ion-exchange column to 
separate amino acids and post-column reaction with ninhydrin/hydrindantin (Beckman Nin-Rx, Beckman Coulter, Fullerton, CA, USA) for detection at 570 and $440 \mathrm{~nm}$. The samples were vortexed, then spun in a microcentrifuge to pellet the solids. A $100 \mu \mathrm{l}$ aliquot of the supernatant was taken from each sample and dried in a Savant SC110A Speed-Vac. The aliquots were dissolved in $100 \mu$ l of loading buffer (Beckman Na-S with 2 nmol homoserine per $100 \mu \mathrm{l}$ as an internal standard), and then filtered. The recovered volume was measured and diluted back up to $100 \mu \mathrm{l}$ to load on the analyzer. The results have been corrected to the original $100 \mu \mathrm{l}$ taken by using the measured volume as the per cent injected.

A collaborating laboratory collected the RD vitreous fluid and performed amino-acid analysis. Calibration curves were constructed using a standard. Analysis of $2 \mu \mathrm{l}$ aliquots began with derivatization of vitreous amino acids with $2 \mu \mathrm{l} 20 \mathrm{mM}$ CBQCA (Molecular Probes, Eugene, OR, USA) in the presence of $2 \mu \mathrm{l} 10 \mathrm{mM}$ cyanide. The $\mathrm{pH}$ value of the vitreous samples was between 2 and 3 due to the acetic acid addition. Therefore, the $10 \mathrm{mM}$ cyanide was diluted in $250 \mathrm{mM}$ phosphate buffer $(\mathrm{pH}$ 12.0) for the derivatization process. The reaction mixture of vitreous sample and CBQCA was allowed to react for $2 \mathrm{~h}$ at room temperature prior to capillary electrophoresis-laser-induced fluorescence (CE-LIF) analysis. ${ }^{36}$ The running buffer consisted of $20 \mathrm{mM}$ sodium tetraborate, $20 \mathrm{mM}$ sodium chloride, $45 \mathrm{mM}$ sodium dodecyl sulphate, and $55 \mathrm{mM} \beta$-cyclodextrin $(\beta-\mathrm{CD})$, and the separation was carried out at $17 \mathrm{kV}$ applied voltage.

The CE-LIF system contained a high-voltage power supply (Spellman, Hauppage, NY, USA), a ZETALIF LIF detector (Picometrics, Paris, France), and an argon ion laser (Coherent, Santa Clara, CA, USA) at $488 \mathrm{~nm}$. All CE analyses were performed at room temperature with $40 \mathrm{~cm} \times 360 \mu \mathrm{m}$ OD $\times 50 \mu \mathrm{m}$ ID (30 $\mathrm{cm}$ effective length) fused-silica capillaries (BioTAQ, Gaithersburg, MD, USA). In this work, gravimetric sample injection was used with a $30 \mathrm{~cm}$ height difference for $5 \mathrm{~s}$. The system operation and data acquisition was performed with a custom LabVIEW (National Instruments, Austin, TX, USA) program and the data were analysed by using Microsoft Excel and Class Eleganza CE station software V5.5 (Ayer Rajah Industrial Estate, Singapore).

\section{Statistical analysis}

Amino-acid concentrations in SRF and RD-Vit were compared with Control-Vit using the Wilcoxon's ranksum test because the data were not normally distributed. Demographic information and clinical characteristics were compared using the two-sample $t$-test, Wilcoxon's rank-sum test, or Fisher's exact test depending on the types of variables (continuous or categorical) and their distributions (normal or non-normal). The association between the concentration of amino acids in the SRF and patient age, sex, affected eye, macular detachment, RD duration, preop vision, extent of $\mathrm{RD}$, presence of grade $\mathrm{C}$ PVR, lens status, and postop vision was assessed either by the Spearman's rank correlation or Wilcoxon's ranksum test. A $P$-value of $<0.05$ was considered statistically significant.

We certify that all applicable institutional and governmental regulations concerning the ethical use of human volunteers were followed during this research.

\section{Results}

Subretinal fluid $(n=20)$ and vitreous fluid $(n=5)$ samples were obtained from patients with primary RD. Vitreous fluid $(n=10)$ from patients with an epiretinal membrane or macular hole served as controls. Basic demographic information and clinical characteristics of the three groups are summarized in Table 1. Mean age was 49.5 years $( \pm 13.8$ years SD) in the SRF group, 66.1 years $( \pm 12.1$ years SD) in the Control-Vit group, and 63.8 years $( \pm 15.5$ years SD) in the RD-Vit group. SRF patients were significantly younger than Control-Vit patients $(P$-value $=<0.01)$. No other significant differences existed in demographic information between the two RD groups and controls. Mean duration of follow-up time was 20.5 months $( \pm 18.7$ months SD, range from 1 week to 75 months) for the SRF group and 4.1 months ( \pm 1.7 months $\mathrm{SD}$, range from 1.5 to 6 months) for RD-Vit. The mean RD

Table 1 Basic demographic information and clinical characteristics

\begin{tabular}{lccc}
\hline & $\begin{array}{c}\text { Vit } \\
\text { Control } \\
(\mathrm{n}=10)\end{array}$ & $\begin{array}{c}\text { RD-Vit } \\
(\mathrm{n}=5)\end{array}$ & $\begin{array}{c}\text { RD-SRF } \\
(\mathrm{n}=20)\end{array}$ \\
\hline Age (years) & & \\
Female & $\mathbf{6 6 . 1 \pm \mathbf { 1 2 . 1 }}$ & $63.8 \pm 15.5$ & $\mathbf{4 9 . 5} \pm \mathbf{1 3 . 8}$ \\
Right eye & $7(70 \%)$ & $5(100 \%)$ & $8(40 \%)$ \\
Pseudophakic & $6(60 \%)$ & $3(60 \%)$ & $10(50 \%)$ \\
Duration of detachment (days) $)^{*}$ & $2(20 \%)$ & $4(80 \%)$ & $6(30 \%)$ \\
Macular detachment & $52.4 \pm 65.4$ & $33.1 \pm 68.9$ \\
Clock hours of retinal & & $4(80 \%)$ & $9(45 \%)$ \\
detachment* & & $6.4 \pm 3.6$ & $5.4 \pm 3.0$ \\
PVR grade C (Y/N) & & 0 & $6(30 \%)$ \\
Preoperative visual acuity & & & \\
Postoperative visual acuity & & $1.4 \pm 0.8$ & $0.9 \pm 0.7$ \\
Duration of follow-up (months) & & $\mathbf{1 . 0} \pm \mathbf{0 . 7}$ & $\mathbf{0 . 4} \pm \mathbf{0 . 5}$ \\
Range of follow-up (months) & $4.1 \pm 1.7$ & $20.5 \pm 18.7$ \\
\hline
\end{tabular}

*Data are presented as mean \pm SD (standard deviation).

RD-SRF, retinal detachment-subretinal fluid.

aSignificantly different between Vit control and RD-SRF $(P$-value $<0.01)$. bSnellen visual acuities were converted into a logarithmic scale ( $\log$ MAR)

'Significantly different between RD-Vit and RD-SRF $(P$-value $=0.03)$. 
Table 2 Amino-acid levels in Vit Control, RD-Vit, and RD-SRF $(\mu \mathrm{M})$

\begin{tabular}{|c|c|c|c|c|c|c|c|c|c|c|}
\hline & Glu & Asp & Gly & Arg & Ala & Tyr & Val & Ile & Leu & Phe \\
\hline $\begin{array}{l}\text { Vit Control } \\
(n=10)\end{array}$ & $1.7 \pm 0.8$ & $1.1 \pm 0.8$ & $4.0 \pm 2.7$ & $39.4 \pm 16.3$ & $88.4 \pm 35.0$ & $31.9 \pm 13.7$ & $112 \pm 4.4$ & $28.3 \pm 12.0$ & $63.5 \pm 21.9$ & $31.7 \pm 11$ \\
\hline $\begin{array}{l}\text { RD Vit } \\
(n=5)\end{array}$ & $13.4 \pm 11.9$ & - & - & - & - & - & - & - & - & - \\
\hline$P$-value ${ }^{a}$ & 0.01 & & & & & & & & & \\
\hline $\begin{array}{l}\text { RD-SRF } \\
(n=20)\end{array}$ & $27.0 \pm 1.7$ & $4.1 \pm 4.0$ & $44.1 \pm 31.0$ & $52.7 \pm 46.0$ & $45.4 \pm 35.7$ & $15.0 \pm 11.1$ & $19.9 \pm 53.8$ & $14.3 \pm 17.9$ & $32.4 \pm 32.1$ & $19.5 \pm 19$ \\
\hline$P$-value ${ }^{\mathrm{b}}$ & $<0.01$ & 0.01 & $<0.01$ & $>0.05$ & $<0.01$ & $<0.01$ & $<0.01$ & $<0.01$ & $<0.01$ & $<0.01$ \\
\hline
\end{tabular}

RD-SRF, retinal detachment-subretinal fluid.

Data are presented as mean \pm SD (standard deviation).

Ala, alanine; Arg, arginine; Asp, aspartate; Glu, glutamate; Gly, glycine; Ile, isoleucine; Leu, leucine; Phe, phenylalanine; Tyr, tyrosine; Val, valine. ${ }^{a}$ Comparison between Vit Control and RD-Vit.

Table 3 Association between glutamate levels of subretinal fluid and characteristics of RD

\begin{tabular}{lcc}
\hline Variable & $\begin{array}{c}\text { Spearman's correlation } \\
\text { coefficients }\end{array}$ & P-value \\
\hline Age (years) & -0.12 & 0.62 \\
Sex (M/F) & -0.12 & 0.60 \\
Affected eye (OD/OS) & -0.17 & 0.46 \\
Macular involvement (Y/N) & 0.08 & 0.74 \\
RD duration (days) & -0.27 & 0.25 \\
Preop vision (logMAR) & 0.13 & 0.59 \\
Extent of RD (\%) & -0.41 & 0.08 \\
PVR (Y/N) grade C & -0.19 & 0.42 \\
Lens status (phakic/ & -0.25 & 0.30 \\
pseudophakic) & & \\
Postop vision (logMAR) & 0.01 & 0.95 \\
Postop vision (logMAR) & 0.27 & 0.42 \\
\hline
\end{tabular}

$\mathrm{RD}$, retinal detachment; logMAR, logarithm of the minimal angle of resolution; OD, Oculus Dexter; OS, Oculus Sinister.

duration was 33.1 days ( \pm 68.9 days $S D$, range from 1 to 270 days) for the SRF group and 52.4 days ( $\pm 65.4 \mathrm{SD}$, range from 3 to 150 days) for the RD-Vit group. A significantly better postoperative visual acuity was observed in the SRF group as compared to the RD-Vit group $(P$-value $=<0.05)$.

Comparisons of mean levels of amino acids between Control-Vit, RD-Vit, and SRF are presented in Table 2. The mean level of glutamate in RD-Vit was $13.4 \mu \mathrm{M}$ $( \pm 11.9 \mu \mathrm{M}$ SD), which was significantly higher than the Control-Vit glutamate level, $1.7 \mu \mathrm{M}( \pm 0.8 \mu \mathrm{M}$ SD, $P$-value $=0.01)$. Glutamate levels in SRF had a mean of $27.0 \mu \mathrm{M}( \pm 19.7 \mu \mathrm{M}$ SD), and were also significantly elevated relative to Control-Vit $(P$-value $<0.01)$.

Aspartate and glycine also had mean concentrations in SRF that were significantly higher than the control group, Control-Vit ( $P$-values: 0.01 and $<0.01$, respectively). Mean arginine levels did not differ significantly between SRF and Control-Vit. All other measured amino acids (alanine, tyrosine, valine, isoleucine, leucine, and phenylalanine) were present in SRF at significantly lower concentrations as compared to Control-Vit $(P$-values all $<0.01)$.

Comparing SRF demographic information and clinical characteristics with glutamate levels in SRF did not yield any significant associations (Table 3 ). However, the data do reveal a significant, positive association between aspartate levels and glutamate levels in SRF (Spearman's correlation coefficient: $0.74, P$-value $=<0.01)$. Glutamate levels in SRF of 11 cases of macula-on detachment did not correlate with postoperative visual acuity (Spearman's correlation coefficient: $0.27, P$-value $=0.42$ ). Tables 4 and 5 show amino-acid concentrations in subretinal and vitreous fluid for individual patients.

\section{Discussion}

Glutamate was present at significantly higher concentrations in SRF and vitreous fluid of patients with primary RD as compared to controls. Subretinal aspartate and glycine levels were also found to be significantly elevated. Furthermore, a significant, positive association was observed between aspartate levels and glutamate levels in SRF.

These findings partially support those of a recent clinical investigation that found elevated glutamate levels in the vitreous of patients with RD. ${ }^{33}$ However, that study, unlike this one, did not show a significant difference in aspartate or glycine levels between RD vitreous and controls. These differences might be explained by variations in high-performance liquid chromatography methods. Another study did find elevated aspartate in cat retina after experimental $\mathrm{RD},{ }^{32}$ and a rat model of retinal ischaemia has shown elevated levels of glycine in the vitreous. ${ }^{27}$ Amino-acid levels in the Control-Vit group were comparable to those found by Honkanen et al. ${ }^{37}$

Several plausible theories exist for the accumulation of glutamate in the retina and vitreous following RD. In 
Table 4 Characteristics of patients with RD and amino-acid level $(\mu \mathrm{M})$ in subretinal or vitreous fluid

\begin{tabular}{|c|c|c|c|c|c|c|c|c|c|c|c|c|c|c|c|c|}
\hline $\begin{array}{l}\text { Age } \\
\text { (years) }\end{array}$ & Sex & $\begin{array}{c}R D \\
\text { (days) }\end{array}$ & Sample & $M a$ & $\begin{array}{l}\text { Preop } \\
\operatorname{VA}(X)\end{array}$ & $\begin{array}{l}\text { Postop } \\
V A(X)\end{array}$ & Glu & $A s p$ & Gly & Arg & Ala & Tyr & Val & Ile & Leu & Phe \\
\hline 44 & $\mathrm{~F}$ & 30 & $S$ & On & 40 & 25 & 23.18 & 3.09 & 30.43 & 20.10 & 47.74 & 10.03 & 2.22 & 4.11 & 24.64 & 15.15 \\
\hline 51 & $\mathrm{M}$ & 5 & S & On & 25 & 20 & 20.39 & 0.56 & 40.90 & 13.80 & 28.86 & 5.15 & 1.41 & 5.13 & 10.11 & 5.68 \\
\hline 56 & $\mathrm{~F}$ & 1 & $S$ & Off & HM & 32 & 55.10 & 11.30 & 145.90 & 148.52 & 48.78 & 10.41 & 223.70 & 80.20 & 147.30 & 77.50 \\
\hline 60 & $\mathrm{M}$ & 1 & $S$ & On & HM & 25 & 23.47 & 1.50 & 23.58 & 45.90 & & 10.52 & 3.61 & 7.83 & 22.65 & 11.04 \\
\hline 58 & $\mathrm{M}$ & 180 & $S$ & Off & CF & 50 & 14.05 & 2.51 & 17.60 & & 26.89 & . & 1.26 & 4.31 & 23.22 & . \\
\hline 63 & $\mathrm{~F}$ & 1 & $S$ & On & 30 & 25 & 10.70 & 1.04 & & 64.61 & 57.91 & 11.07 & 2.78 & 9.14 & & 11.40 \\
\hline 55 & $\mathrm{~F}$ & 2 & S & On & 20 & 32 & 48.90 & 3.39 & 34.20 & & 47.22 & 7.38 & 1.84 & 7.41 & 18.69 & 8.89 \\
\hline 72 & $\mathrm{M}$ & 14 & $S$ & Off & HM & $\mathrm{CF}$ & 2.22 & 0.79 & 33.90 & 47.07 & 10.73 & 41.42 & 13.68 & 33.36 & 55.34 & 38.98 \\
\hline 45 & $\mathrm{~F}$ & 30 & S & On & 20 & 20 & 1.25 & 0.60 & 32.90 & & 13.08 & 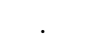 & . & . & & . \\
\hline 76 & $\mathrm{~F}$ & 7 & $S$ & Off & $\mathrm{CF}$ & 25 & 30.12 & 5.90 & 69.21 & 35.26 & 84.30 & 12.86 & 3.49 & 14.16 & 28.44 & 15.65 \\
\hline 64 & $\mathrm{M}$ & 7 & S & Off & 200 & 63 & 16.09 & 3.70 & 17.23 & 13.46 & 11.56 & 2.34 & 0.63 & 1.29 & 6.42 & 2.24 \\
\hline 34 & $\mathrm{M}$ & 5 & S & On & 160 & 25 & 2.10 & 0.52 & 82.20 & 53.12 & 51.73 & 15.28 & 4.70 & 13.01 & 26.19 & 15.06 \\
\hline 46 & $\mathrm{M}$ & 21 & $S$ & Off & 160 & 200 & 9.84 & 2.05 & 46.70 & 18.09 & 21.65 & 4.24 & 1.17 & 2.71 & 5.78 & 20.90 \\
\hline 50 & $\mathrm{M}$ & 1 & $S$ & On & 40 & 20 & 47.42 & 2.53 & 44.49 & 60.41 & 12.91 & 21.11 & 5.85 & 14.44 & 47.84 & 20.40 \\
\hline 33 & $\mathrm{~F}$ & 60 & $S$ & Off & $\mathrm{CF}$ & 200 & 30.12 & 6.45 & 24.12 & 70.38 & 65.67 & 22.51 & 5.27 & 13.65 & 30.24 & 11.62 \\
\hline 33 & $\mathrm{M}$ & 270 & $S$ & Off & 400 & $\mathrm{CF}$ & 30.12 & 6.45 & 24.65 & 71.38 & 65.65 & & 5.27 & 13.33 & 30.65 & 11.65 \\
\hline 24 & $\mathrm{M}$ & 1 & $S$ & On & 30 & 20 & 21.81 & 4.80 & 15.07 & 26.72 & 40.15 & 8.98 & 2.51 & 6.05 & 17.28 & 7.42 \\
\hline 41 & $\mathrm{~F}$ & 3 & $S$ & On & 40 & 20 & 26.13 & 4.25 & 31.47 & 25.77 & 43.51 & 8.17 & 2.42 & 7.65 & 15.29 & 7.68 \\
\hline 48 & $\mathrm{M}$ & 1 & $S$ & On & 200 & 25 & 71.70 & 16.70 & 56.80 & 173.90 & 164.30 & 29.00 & 76.60 & 27.70 & 55.00 & 15.90 \\
\hline 36 & $\mathrm{M}$ & 21 & S & Off & 40 & 30 & 56.17 & 2.89 & 66.80 & 7.66 & 20.82 & 34.99 & . & 5.29 & 18.59 & 54.43 \\
\hline 66 & $\mathrm{~F}$ & & V & Off & & & 11.49 & & & & & & & & & \\
\hline 77 & $\mathrm{~F}$ & & V & Off & & & 2.49 & & & & & & & & & \\
\hline 34 & $\mathrm{~F}$ & & $\mathrm{~V}$ & On & & & 29.97 & & & & & & & & & \\
\hline 72 & $\mathrm{~F}$ & & $\mathrm{~V}$ & Off & & & 20.44 & & & & & & & & & \\
\hline 70 & $\mathrm{~F}$ & & V & Off & & & 2.63 & & & & & & & & & \\
\hline
\end{tabular}

Ala, alanine; Arg, arginine; Asp, aspartate; Glu, glutamate; Gly, glycine; Ile, isoleucine; Leu, leucine; Ma, macular status; Phe, phenylalanine; Postop VA, postoperative visual acuity (20/X); Preop VA, preoperative visual acuity (20/X); RD, retinal detachment; S, subretinal fluid; Tyr, tyrosine; V, vitreous; Val, valine; HM, Hand Motions; CF, Counting Fingers (data not avilable).

Table 5 Characteristics of control patients and amino-acid level $(\mu \mathrm{M})$ in vitreous fluid

\begin{tabular}{|c|c|c|c|c|c|c|c|c|c|c|c|c|}
\hline Age (years) & Sex & Disease & Glu & Asp & Gly & Arg & Ala & Tyr & Val & Ile & Leu & Phe \\
\hline 66 & $\mathrm{~F}$ & ERM & 2.92 & 1.45 & 8.84 & 40.89 & 103.46 & 31.97 & 108.76 & 25.41 & 61.22 & 28.95 \\
\hline 79 & $\mathrm{~F}$ & ERM & 1.12 & 0.45 & 3.95 & 36.85 & 55.90 & 14.67 & 63.64 & 19.03 & 40.73 & 15.76 \\
\hline 41 & $\mathrm{~F}$ & ERM & 0.70 & 0.80 & 5.05 & 34.00 & 68.81 & 34.03 & 87.51 & 19.86 & 51.07 & 33.60 \\
\hline 67 & M & ERM & 1.68 & 2.63 & 2.68 & 55.83 & 104.48 & 30.70 & 118.43 & 29.32 & 65.22 & 43.36 \\
\hline 80 & $\mathrm{~F}$ & ERM & 1.47 & 1.78 & 8.48 & 63.91 & 169.40 & 34.50 & 197.37 & 44.09 & 96.34 & 46.34 \\
\hline 63 & $\mathrm{~F}$ & ERM & 2.39 & 1.00 & 2.06 & 24.39 & 76.85 & 26.64 & 70.82 & 14.86 & 41.71 & 20.89 \\
\hline 64 & M & ERM & 2.93 & 1.67 & 2.30 & 41.16 & 105.25 & 66.22 & 177.07 & 50.41 & 104.33 & 47.02 \\
\hline 77 & $\mathrm{~F}$ & $\mathrm{MH}$ & 1.80 & 0.21 & 2.35 & 5.75 & 86.31 & 23.11 & 77.04 & 18.19 & 44.21 & 25.60 \\
\hline 71 & $\mathrm{M}$ & ERM & 1.37 & 0.26 & 2.12 & 40.20 & 50.81 & 22.65 & 111.22 & 37.74 & 69.79 & 25.61 \\
\hline 53 & $\mathrm{~F}$ & $\mathrm{MH}$ & 1.09 & 0.76 & 1.90 & 50.70 & 62.43 & 35.37 & 108.98 & 23.80 & 60.75 & 30.24 \\
\hline
\end{tabular}

Ala, alanine; Arg, arginine; Asp, aspartate; ERM, epiretinal membrane; Glu, glutamate; Gly, glycine; Ile, isoleucine; Leu, leucine; MH, macular hole; Phe, phenylalanine; Tyr, tyrosine; Val, valine.

attached retina, glutamate is released from presynaptic neurons into the extracellular space, from which most of it is transported into Müller cells via the glutamate/ aspartate transporter. ${ }^{38}$ Glutamate is immediately converted into the non-toxic amino-acid glutamine by the enzyme GS, and then exported to neighbouring neurons where it is hydrolysed by glutaminase to reform glutamate. ${ }^{39-41}$ Disruption of the retinal glutamate reuptake transporter, glutamate/aspartate transporter, has been shown to lead to increased vitreal glutamate and subsequent ganglion cell death in an animal study. ${ }^{42}$ Glutamate/aspartate transporter-deficient mice show a much greater sensitivity to the retinal damage induced by ischaemia. ${ }^{43}$ Ischaemic disruption of glutamate transport could be caused by elevated extracellular levels of $\mathrm{K}^{+} .{ }^{44}$ Studies of experimental RD in cat found 
elevated levels of glutamate coupled with a rapid decrease in GS expression in Müller cells. ${ }^{14,30}$ Marc et al ${ }^{30}$ found that glutamate levels persisted in Müller cells following RD but decreased in the retinal pigment epithelium, a pattern that suggests an alteration in glutamate metabolism rather than a sustained increase in neuronal glutamate release. GS has also been shown to influence glutamate clearance from the synaptic cleft and amplified expression of GS has provided protection from excess glutamate. ${ }^{45,46}$ Retinal ischaemia secondary to RD may play a role in disrupting the mechanisms of reuptake and degradation responsible for maintaining glutamate homeostasis.

Glutamate toxicity most probably results from osmotic damage and calcium insult. Excess sodium entry into the cell, stimulated by elevated glutamate levels, is coupled with water and chloride entry, which leads to osmotic cell lysis and neuronal swelling. ${ }^{47}$ In addition, glutamate has a high affinity for $N$-methyl-D-aspartate (NMDA) receptors, overactivation of which causes a large influx of calcium into the cell. This increase in calcium leads to protease and lipase activation, endonuclease activation, kinase activation, and cell membrane deterioration, and ultimately contributes to cell death. Glutamate-mediated NMDA stimulation may also lead to increased nitric oxide production, with a resultant inhibitory effect on energy production in the mitochondria. ${ }^{27}$

Because aspartate is also regulated by glutamate/ aspartate transporter, the reduced reuptake associated with ischaemia could also account for the increased concentration of this amino acid after RD. ${ }^{48}$ Increased neuronal release of aspartate under ischaemic conditions has also been found. Altered glutamate metabolism and transamination reactions associated with ischaemia could also account for the elevated levels of aspartate, which is a metabolite/precursor for glutamate. ${ }^{49}$ Both of these explanations can account for the observed positive association between aspartate and glutamate levels in SRF. Aspartate may also act as a neurotoxin, ${ }^{50}$ acting through NMDA receptors in a fashion similar to glutamate.

Elevated levels of glycine following RD may result from ischaemic reversal of the glycine transporter, found in Müller cells. ${ }^{51}$ Since glycine also acts on NMDA receptors and is thought to be required for channel activation by glutamate, it is possible that this amino acid contributes to some of the toxicity observed after RD.

Analysis of demographic information revealed a significantly older Vit-Control population as compared to the SRF group. This finding is likely due to the fact that the control group was composed of patients with macular hole or epiretinal membrane, ocular conditions that typically affect older individuals. However, there was no significant difference in age between the RD-Vit and the Control-Vit groups. This may be due to the fact that the RD-Vit population was disproportionately composed of patients who were pseudophakic at the time of their RD, making the choice of vitrectomy more likely. The data show significantly better postoperative visual acuity in the SRF group as compared to RD-Vit group. This finding is attributable to the fact that the RD-Vit group possessed a preponderance of those clinical characteristics that are associated with poor visual outcome: lower mean preoperative visual acuity, increased percentage of patients with macula-off detachment, and increased mean duration of RD compared to the SRF group. No correlation was observed between SRF glutamate levels and pre or postoperative visual acuity, either overall or in patients with macula-on RD. However, the small number of patients in this study limits our ability to detect such a correlation, and a larger study will be needed to determine whether there exists a relationship between glutamate levels and visual impairment following RD. Although the mean duration of RD was shorter for the SRF group as compared to the RD-Vit group, mean glutamate levels were higher in the SRF group. Glutamate may concentrate more in the smaller subretinal space as compared to the larger vitreous space. Larger studies comprised of groups with paired duration of RD are needed to further evaluate this finding.

There are some limitations to this study that should be kept in mind when interpreting these results. Compared to the study by Diederen et al, ${ }^{33}$ this study analysed relatively few patients. This small sample size may explain the observed lack of association between glutamate levels and pre and postoperative visual acuity. This study lacked an ideal SRF control sample for comparison due to the impossibility of collecting SRF in patients without RD. However, the Control-Vit fluid obtained nevertheless offers an appropriate comparison since SRF is initially composed of liquid vitreous. The two different methods used to measure amino-acid levels will likely yield slightly different means and standard deviations. The extent of this difference; however, is not likely significant. Comparison of vitreous controls analysed with both methods yielded similar results. Indeed, using two different techniques, provided their results are similar, reduces the likelihood of a bias in either technique going undetected.

Oxygen supplementation may be effective in renormalizing the glutamate cycle and preventing further excitoxicity seen after RD. In a study by Lewis et $a l^{24}$ a cat model of RD was placed in a hyperoxic environment and showed a stabilization of glutamaterelated neurochemical signatures. NMDA receptor antagonists, such as memantine and dextromethorphan, may also prove to be beneficial in attenuating retinal cell 
death secondary to glutamate excitotoxicity. ${ }^{19,27}$ Animal studies on the use of NMDA receptor antagonists in RD are needed to investigate these drugs as a possible therapeutic modality.

In conclusion, this study found increased levels of glutamate, glycine, and aspartate in SRF, and of glutamate in vitreous fluid of patients with primary rhegmatogenous RD. This study also found a significant, positive association between glutamate and aspartate in SRF. Our data do not show an association between any other amino-acid levels and clinical characteristics collected in this study, including pre and postoperative visual acuity. This study lends further support to the hypothesis that elevated glutamate levels in the vitreous and SRF may result from ischaemia secondary to RD, expanding upon earlier animal models ${ }^{22,30-32}$ of RD and a recent clinical study of amino-acid levels in vitreous fluid of patients with RD. ${ }^{33}$ To our knowledge, this is the first study that has demonstrated elevated levels of glutamate in SRF following RD. Additional studies are needed to delineate the role of glutamate in human RD and to investigate further any possible association between glutamate levels and visual acuity.

\section{Acknowledgements}

Amino-acid analyses of SRF and control vitreous fluid were done at the WM Keck Foundation Biotechnology Resource Laboratory of Yale University (New Haven, CT, USA). Retinal detachment vitreous fluid was analysed in the Department of Chemistry, University of Illinois at Chicago (Chicago, IL, USA). M-JL, JSP, and SAS gratefully acknowledge financial support from NIH EY014908. The authors declare that they have no proprietary interest in this research. Dr Arroyo is the recipient of an NIH K-23 Physician Training Award. This work was also supported by the Grmstraw-Code WSCZ Charitable Foundation.

\section{References}

1 Tani P, Robertson DM, Langworthy A. Rhegmatogenous retinal detachment without macular involvement treated with scleral buckling. Am J Ophthalmol 1980; 90(4): 503-508.

2 Ross WH. Visual recovery after macula-off retinal detachment. Eye 2002; 16(4): 440-446.

3 Anderson DH, Stern WH, Fisher SK, Erickson PA, Borgula GA. Retinal detachment in the cat: the pigment epithelialphotoreceptor interface. Invest Ophthalmol Vis Sci 1983; 24(7): 906-926.

4 Machemer R. Experimental retinal detachment in the owl monkey. IV. The reattached retina. Am J Ophthalmol 1968; 66(6): 1075-1091.

5 Kroll AJ, Machemer R. Experimental retinal detachment in the owl monkey.V. Electron microscopy of the reattached retina. Am J Ophthalmol 1969; 67(1): 117-130.
6 Kroll AJ, Machemer R. Experimental retinal detachment and reattachment in the rhesus monkey. Electron microscopic comparison of rods and cones. Am J Ophthalmol 1969; 68(1): 58-77.

7 Guerin CJ, Anderson DH, Fariss RN, Fisher SK. Retinal reattachment of the primate macula. Photoreceptor recovery after short-term detachment. Invest Ophthalmol Vis Sci 1989; 30(8): 1708-1725.

8 Arroyo JG, Yang L, Bula D, Chen DF. Photoreceptor apoptosis in human retinal detachment. Am J Ophthalmol 2005; 139(4): 605-610.

9 Erickson PA, Fisher SK, Anderson DH, Stern WH, Borgula GA. Retinal detachment in the cat: the outer nuclear and outer plexiform layers. Invest Ophthalmol Vis Sci 1983; 24(7): 927-942.

10 Yang L, Bula D, Arroyo JG, Chen DF. Preventing retinal detachment-associated photoreceptor cell loss in Baxdeficient mice. Invest Ophthalmol Vis Sci 2004; 45(2): 648-654.

11 Enoch JM, Van Loo Jr JA, Okun E. Realignment of photoreceptors disturbed in orientation secondary to retinal detachment. Invest Ophthalmol 1973; 12(11): 849-853.

12 Fitzgerald CR, Birch DG, Enoch JM. Functional analysis of vision in patients after retinal detachment repair. Arch Ophthalmol 1980; 98(7): 1237-1244.

13 Geller SF, Lewis GP, Fisher SK. FGFR1, signaling, and AP-1 expression after retinal detachment: reactive Muller and RPE cells. Invest Ophthalmol Vis Sci 2001; 42(6): 1363-1369.

14 Lewis GP, Guerin CJ, Anderson DH, Matsumoto B, Fisher SK. Rapid changes in the expression of glial cell proteins caused by experimental retinal detachment. Am J Ophthalmol 1994; 118(3): 368-376.

15 Uhlmann S, Bringmann A, Uckermann O, Pannicke T, Weick M, Ulbricht E et al. Early glial cell reactivity in experimental retinal detachment: effect of suramin. Invest Ophthalmol Vis Sci 2003; 44(9): 4114-4122.

16 Iandiev I, Uckermann O, Pannicke Tet al. Glial cell reactivity in a porcine model of retinal detachment. Invest Ophthalmol Vis Sci 2006; 47(5): 2161-2171.

17 Fisher SK, Erickson PA, Lewis GP, Anderson DH. Intraretinal proliferation induced by retinal detachment. Invest Ophthalmol Vis Sci 1991; 32(6): 1739-1748.

18 Lewis GP, Linberg KA, Fisher SK. Neurite outgrowth from bipolar and horizontal cells after experimental retinal detachment. Invest Ophthalmol Vis Sci 1998; 39(2): 424-434.

19 Calzada JI, Jones BE, Netland PA, Johnson DA. Glutamateinduced excitotoxicity in retina: neuroprotection with receptor antagonist, dextromethorphan, but not with calcium channel blockers. Neurochem Res 2002; 27(1-2): 79-88.

20 Olney JW. Glutamate-induced retinal degeneration in neonatal mice: electron microscopy of the acutely evolving lesion. J Neuropathol Exp Neurol 1969; 28(3): 455-474.

21 Lucas DR, Newhouse JP. The toxic effect of sodium Lglutamate on the inner layers of the retina. AMA Arch Ophthalmol 1957; 58(2): 193-201.

22 Sherry DM, Townes-Anderson E. Rapid glutamatergic alterations in the neural retina induced by retinal detachment. Invest Ophthalmol Vis Sci 2000; 41(9): 2779-2790.

23 Sisk DR, Kuwabara T. Histologic changes in the inner retina of albino rats following intravitreal injection of monosodium L-glutamate. Graefes Arch Clin Exp Ophthalmol 1985; 223(5): 250-258.

24 Lewis G, Mervin K, Valter K, Maslim J, Kappel PJ, Stone J et al. Limiting the proliferation and reactivity of retinal Muller cells during experimental retinal detachment: the 
value of oxygen supplementation. Am J Ophthalmol 1999; 128(2): 165-172.

25 Mervin K, Valter K, Maslim J, Lewis G, Fisher S, Stone J. Limiting photoreceptor death and deconstruction during experimental retinal detachment: the value of oxygen supplementation. Am J Ophthalmol 1999; 128(2): 155-164.

26 Louzada-Junior P, Dias JJ, Santos WF, Lachat JJ, Bradford HF, Coutinho-Netto J et al. Glutamate release in experimental ischaemia of the retina: an approach using microdialysis. J Neurochem 1992; 59(1): 358-363.

27 Lagreze WA, Knorle R, Bach M, Feuerstein TJ. Memantine is neuroprotective in a rat model of pressure-induced retinal ischemia. Invest Ophthalmol Vis Sci 1998; 39(6): 1063-1066.

28 Napper GA, Pianta MJ, Kalloniatis M. Localization of amino acid neurotransmitters following in vitro ischemia and anoxia in the rat retina. Vis Neurosci 2001; 18(3): 413-427.

29 Napper GA, Kalloniatis M. Neurochemical changes following postmortem ischemia in the rat retina. Vis Neurosci 1999; 16(6): 1169-1180.

30 Marc RE, Murry RF, Fisher SK, Linberg KA, Lewis GP. Amino acid signatures in the detached cat retina. Invest Ophthalmol Vis Sci 1998; 39(9): 1694-1702.

31 Sasoh M, Ma N, Yoshida S, Semba R, Uji Y. Immunocytochemical localization of glutamate in normal and detached cat retina. Invest Ophthalmol Vis Sci 1998; 39(5) 786-792.

32 Sasoh M, Ma N, Ito Y, Esaki K, Uji Y. Changes in localization of amino acids in the detached cat retina. Ophthalmic Res 2006; 38(2): 74-82.

33 Diederen RM, La Heij EC, Deutz NE, Kijlstra A, Kessels AG van Eijk HM et al. Increased glutamate levels in the vitreous of patients with retinal detachment. Exp Eye Res 2006; 83(1): 45-50.

34 Jaffe GJ, Brownlow R, Hines J. Modified external needle drainage procedure for rhegmatogenous retinal detachment. Retina 2003; 23(1): 80-85.

35 Arroyo JG, Bula DV, Yang L, Chen DF. Retinal biopsy techniques for the removal of retinal tissue fragments. Ophthalmic Surg Lasers Imaging 2005; 36(1): 76-78.

36 Thongkhao-On K, Kottegoda S, Pulido JS, Shippy SA. Determination of amino acids in rat vitreous perfusates by capillary electrophoresis. Electrophoresis 2004; 25(17): 2978-2984.

37 Honkanen RA, Baruah S, Zimmerman MB, Khanna CL Weaver YK, Narkiewicz J et al. Vitreous amino acid concentrations in patients with glaucoma undergoing vitrectomy. Arch Ophthalmol 2003; 121(2): 183-188.
38 Pow DV, Barnett NL, Penfold P. Are neuronal transporters relevant in retinal glutamate homeostasis? Neurochem Int 2000; 37(2-3): 191-198.

39 Pow DV, Crook DK. Direct immunocytochemical evidence for the transfer of glutamine from glial cells to neurons: use of specific antibodies directed against the Dstereoisomers of glutamate and glutamine. Neuroscience 1996; 70(1): 295-302.

40 Van den Berg CJ. Glutamate and glutamine, in Handbook in Neurochemistry. In: A Lajtha (ed). Plenum Press: New York, 1970, pp 355-379.

41 Kennedy AJ, Voaden MJ, Marshall J. Glutamate metabolism in the frog retina. Nature 1974; 252(5478): 50-52.

42 Vorwerk CK, Naskar R, Schuettauf F, Quinto K, Zurakowski $\mathrm{D}$, Gochenauer G et al. Depression of retinal glutamate transporter function leads to elevated intravitreal glutamate levels and ganglion cell death. Invest Ophthalmol Vis Sci 2000; 41(11): 3615-3621.

43 Harada T, Harada C, Watanabe M, Inoue Y, Sakagawa T, Nakayama $\mathrm{N}$ et al. Functions of the two glutamate transporters GLAST and GLT-1 in the retina. Proc Natl Acad Sci USA 1998; 95(8): 4663-4666.

44 Napper GA, Pianta MJ, Kalloniatis M. Reduced glutamate uptake by retinal glial cells under ischemic/hypoxic conditions. Vis Neurosci 1999; 16(1): 149-158.

45 Shaked I, Ben-Dror I, Vardimon L. Glutamine synthetase enhances the clearance of extracellular glutamate by the neural retina. J Neurochem 2002; 83(3): 574-580.

46 Gorovits R, Avidan N, Avisar N, Shaked I, Vardimon L. Glutamine synthetase protects against neuronal degeneration in injured retinal tissue. Proc Natl Acad Sci USA 1997; 94(13): 7024-7029.

47 Werth JL, Park TS, Silbergeld DL, Rothman SM. Excitotoxic swelling occurs in oxygen and glucose deprived human cortical slices. Brain Res 1998; 782(1-2): 248-254.

48 Marcaggi P, Hirji N, Attwell D. Release of L-aspartate by reversal of glutamate transporters. Neuropharmacology 2005; 49(6): 843-849.

49 Kalloniatis M, Tomisich G. Amino acid neurochemistry of the vertebrate retina. Prog Retin Eye Res 1999; 18(6): 811-866.

50 Saransaari P, Oja SS. Mechanisms of D-aspartate release under ischemic conditions in mouse hippocampal slices. Neurochem Res 1999; 24(8): 1009-1016.

51 Phillis JW, O'Regan MH. Characterization of modes of release of amino acids in the ischemic/reperfused rat cerebral cortex. Neurochem Int 2003; 43(4-5): 461-467. 